\title{
A single case report of Vulval Stenosis and its management
}

\author{
Bowa $\mathrm{K}^{1 *}$, Chalwe $\mathrm{M}^{2}$ and Kandonga $\mathrm{B}^{3}$ \\ ${ }^{1}$ Professor of Urology Michael Chilufya Sata Copperbelt University School of Medicine, Ndola, Zambia \\ ${ }^{2}$ Senior Registrar Arthur Davison Children's Hospital and Ndola Teaching Hospital, Ndola, Zambia \\ ${ }^{3}$ Registrar Ndola Teaching Hospital, Zambia
}

\section{Case Report}

A 6 month female old child was brought to the Arthur Davison Children's hospital by her mother with the complaint that the child had no vaginal opening. On a careful history it was discovered that the child was born without a vaginal opening. The mother explained that the child passes urine without pain or difficulty. The child was born at the local clinic, where the mother was assured that the vaginal opening would develop as the child got older. She had become concerned when she observed no improvement as the child grew older, and she returned to her local clinic. The local clinic referred her to the Arthur Davison Children's Hospital, which is the National Children's referral hospital in Zambia.

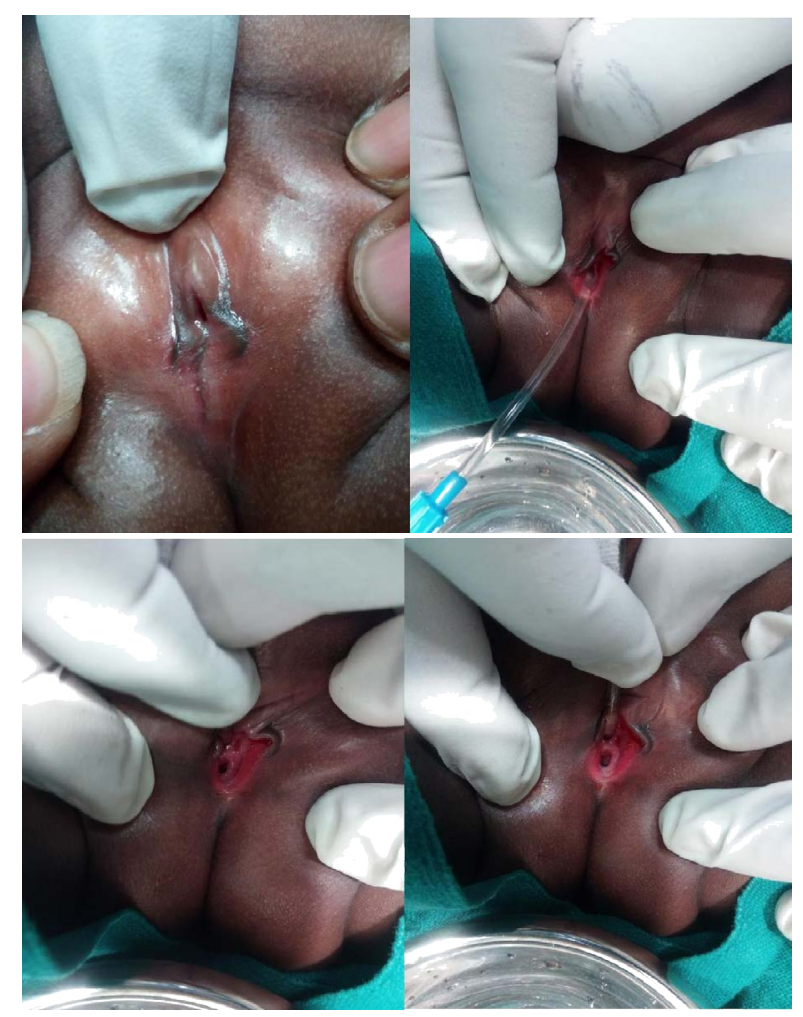

Copyright: (C2018 Schalow G. This is an open-access article distributed under the terms of the Creative Commons Attribution License, which permits unrestricted use, distribution, and reproduction in any medium, provided the original author and source are credited.
The child was examined in the paediatric urology clinic. She was a healthy baby girl who weight $7 \mathrm{~kg}$ in weight. Her general physical examination was normal. The external genitalia showed a normal clitoris with a skin hood. The labia minora though present where adherent to each other in the lower midline. The urethral meatus was visible below the clitoris. The Labia Majora were absent and there was a midline pigment ridge where the Vestibule of the Vulva would have been. The vaginal opening was also absent. A diagnosis of Vulva Stenosis with Vaginal Atresia was made.

The child was book on the next theatre list for an Examination under Anaesthesia to assess the extent of the problem and to plan for appropriate management.

The child was given general anaesthesia and placed supine. Using a 6 French small semi rigid feeding tube the labia minora and majora where separate in the midline alone the midline pigmented rigid. The two labia had soft adhesions which were easily broken with minimal bleeding by the tube. The vestibule, hymen and vagina where seen clearly after this manoeuvre.

A review of the literature shows that though congenital Vaginal Stenosis has been described they are very few reported cases of Congenital Vulva stenosis [1]. A number of acquired cases are described mainly secondary to female genital mutilation in the countries around the Horn of Africa [2]. A few cases are described which are related to skin diseases such as lichen sclerosis and Paget's disease [3,4]. It is suggested by this experience that this condition may easily be resolved by gently breaking the adhesions across the labia with a soft tube under anaesthesia.

\section{References}

1. Kringel U (1950) Congenital valvular stenosis of the female urethra. Zentralbl Gynakol 72: 1443-51. [Crossref]

2. Dirie MA, Lindmark G (1991) A hospital study of the complications of female circumcision. Trop Doct 21: 146-8. [Crossref]

3. Singh S, De D, Saikia UN, Kanwar AJ (2013) Lichen planus causing severe vulva stenosis: rare presentation of a common dermatosis. Int J Dermatol 52: 1447-9. [Crossref]

4. Damiani GR, Loverro G, Tagliabue R, Riva D, Gaetani M, et al. (2012) Recurrence of Paget's disease: total vaginal occlusion and partial occlusion of urethra. Acta Obstet Gynecol Scand 91: 1125-6. [Crossref]

*Correspondence to: Bowa K, Professor of Urology, Copperbelt University School of Medicine, Ndola, Zambia, Tel: 260978157961; E-mail: kbowa@yahoo.com

Received: August 13, 2018; Accepted: August 22, 2018; Published: August 28, 2018 